This item was submitted to Loughborough's Research Repository by the author.

Items in Figshare are protected by copyright, with all rights reserved, unless otherwise indicated.

\title{
Commensurate-incommensurate transition and domain wall dynamics of adsorbed overlayers on a honeycomb substrate
}

PLEASE CITE THE PUBLISHED VERSION

https://doi.org/10.1209/0295-5075/116/56002

PUBLISHER

(C) EPLA

VERSION

AM (Accepted Manuscript)

\section{PUBLISHER STATEMENT}

This work is made available according to the conditions of the Creative Commons Attribution-NonCommercialNoDerivatives 4.0 International (CC BY-NC-ND 4.0) licence. Full details of this licence are available at: https://creativecommons.org/licenses/by-nc-nd/4.0/

\section{LICENCE}

CC BY-NC-ND 4.0

\section{REPOSITORY RECORD}

Elder, Ken R., C.V. Achim, E. Granato, S.C. Ying, and Tapio Ala-Nissila. 2019. "Commensurateincommensurate Transition and Domain Wall Dynamics of Adsorbed Overlayers on a Honeycomb Substrate". figshare. https://hdl.handle.net/2134/28172. 


\title{
Commensurate-Incommensurate Transition and Domain-Wall Dy- namics of Adsorbed Overlayers on a Honeycomb Substrate
}

\author{
K.R. Elder ${ }^{1,2}$, C.V. Achim ${ }^{3}$, E. Granato ${ }^{4,5}$, S.C. Ying ${ }^{5}$ and T. Ala-Nissila ${ }^{2,5}$ \\ 1 Department of Physics, Oakland University - Rochester, Michigan 48309-4487, USA \\ 2 Department of Applied Physics, Aalto University School of Science, P.O. Box 11000 - FI-00076 Aalto, Espoo, \\ Finland \\ 3 Water Research Center for Agriculture and Mining (CRHIAM), University of Concepcin, Chile \\ 4 Laboratório Associado de Sensores e Materiais, Instituto Nacional de Pesquisas Espaciais - 12227-010 São José dos \\ Campos, SP, Brazil \\ 5 Department of Physics, P.O. Box 1843, Brown University - Providence, RI 02912-1843, USA
}

\begin{abstract}
PACS 68.43. Jk - Diffusion of adsorbates, kinetics of coarsening and aggregation
PACS 68.35.Gy - Mechanical properties; surface strains

PACS 68.43. De - Statistical mechanics of adsorbates
\end{abstract}

\begin{abstract}
We introduce an effective one-mode Phase-field Crystal model for studying the commensurate-incommensurate transition and domain wall dynamics of the $(\sqrt{3} \times \sqrt{3}) R 30^{\circ}$ phase found in systems such as $\mathrm{Xe} / \mathrm{Pt}(111)$, or $\mathrm{Xe}$ and $\mathrm{Kr}$ on Graphite. The model allows us to study large systems where the domain walls can be separated over large macroscopic distances and at same time incorporate the microscopic details of the domain wall structures. The resulting phase diagram shows that an intermediate stripe incommensurate phase always separates the commensurate phase from the honeycomb incommensurate phases. The energy of the domain wall crossing is investigated. We also find that near a step edge, the domain walls tend to align perpendicular to the step edge, in agreement with recent experimental observations.
\end{abstract}

In many adsorption systems the maxima (minima) of the adsorption potential due to the substrate form a honeycomb (triangular) lattice [1]. This has been referred to previously as a honeycomb substrate [2]. Examples of such systems, which have been investigated in detail, are Xe on graphite [3], Kr on graphite [4] and Xe/Pt(111) [5]. For these adsorption systems, the ideal separation between the adsorbate atoms is close to $\sqrt{3} a$, where $a$ is the distance between neighboring adsorption potential minimum. For small strain energies, the lowest energy commensurate states is the one that adatoms occupy only a third of the minima, forming the commensurate $(\sqrt{3} \times \sqrt{3}) R 30^{\circ}$ phase. Experimentally, an apparent continuous phase transition from the commensurate $(\sqrt{3} \times \sqrt{3}) R 30^{\circ}$ to an incommensurate phase with a honeycomb network of domain walls (HoI phase) has been observed for $\mathrm{Kr}$ and Xe on Graphite as function of temperature and coverage. In addition, for $\mathrm{Xe} / \mathrm{Pt}(111)$ another uniaxial symmetry incommensurate phase with a striped array of domain walls has been observed $[3,5]$.

Several theoretical works [6-8], however, have deter- mined that the transition from the commensurate $(\sqrt{3} \times$ $\sqrt{3}) R 30^{\circ}$ to the HoI phase should be first order. Bak et al. [7] argued that the symmetry of the domain wall patterns in the incommensurate phase is determined by the energy of the domain wall crossing. For a negative value of domain wall crossing energy, the HoI phase is favored rather than a parallel set of striped domain wall (SI) phase. This theory predicts that in this case a discontinuous transition from commensurate phase to a HoI phase should take place. For positive domain-wall crossing energy, the number of crossings must be as small as possible and the transition of the commensurate phase into SI phase is favored. This transition is predicted to be continuous with the distance between the walls diverging logarithmically as one approaches the critical point. This is followed by a first-order transition from the SI phase to the HoI phase. Note that the terminology of stripe, honeycomb used here refers to the symmetry of the domain wall pattern separating the commensurate domains and not the lattice joining the center of domains that has also been used in the literature [9]. 
Although these theoretical arguments about the relation of energy of the domain wall crossing to the nature of the actual incommensurate phases are quite general, they are based on a mean field theory that ignore thermal fluctuations and wall thickness. Even within mean field theory, only the SI phase is well understood, while the domain wall crossing energy and the details of the two dimensional honeycomb network of domain walls and its energetics have not been fully investigated analytically. In addition computational efforts using molecular dynamics have been hindered by the large length scales required for examining the commensurate-incommensurate transition. In this work, we will examine these issues using an amplitude representation of the phase field crystal formalism [11] which allows us to study the microscopic details of the domain wall crossings and the HoI and SI phases, yet allowing the sample size to be macroscopic $[9,10]$ which is particularly important near the onset of the commensurate-incommensurate transition.

It has been shown $[2,9-11]$ that instead of the fully microscopic atomic model, a useful theoretical formalism for studying the different phases in adsorption systems and the transition between them is the phase field crystal (PFC) model. In this model, the free energy of the adsorption system is described by the functional

$$
\begin{aligned}
F[n(\vec{r})] & =\int d \vec{r}\left\{\frac{n(\vec{r})}{2}\left[\Delta B+B_{x}\left(q_{0}^{2}+\nabla^{2}\right)^{2}\right] n(\vec{r})(1)\right. \\
& \left.-\frac{t}{3} n(\vec{r})^{3}+\frac{v}{4} n(\vec{r})^{4}+V(\vec{r}) n(\vec{r})\right\} .
\end{aligned}
$$

where $V(\vec{r})$ is the periodic adsorption potential due to the adatom substrate interaction with a lattice constant $a$ as depicted in Fig. 1a, and $n(r)$ is a coarse grained dimensionless density field measured from the average density $\bar{n}$ characterizing the real space configuration of the adsorbate film. The adatoms form a hexagonal phase in the absence of the substrate potential with lattice constant $a_{f}=4 \pi / \sqrt{3} q_{0}$ and the commensurate $\sqrt{3} \times \sqrt{3} R 30^{\circ}$ phase has lattice constant $a_{c}=\sqrt{3} a$. The lattice misfit parameter is defined as $\epsilon=\left(a_{c}-a_{f}\right) / a_{c}$. We are interested in the various phases which can occur due to the competition of the strain energy of the film resulting from the lattice mismatch and the potential coupling between substrate and film. The equilibrium density $n(\vec{r})$ is determined by minimizing the free-energy functional in Eq.(1). However, solving for the minimum free energy phase using the full PFC model is limited to relative small size systems because of the fine-grid mesh required to resolve all the atomic scale details of the various phases. In the past, an alternate amplitude expansion has been developed to overcome this problem $[9,10,12-17]$.

When applying this formalism to the adsorption system, it is useful to expand the density in terms of the commensurate sublattices states that are depicted in Fig. 1b, i.e.,

$$
n(\vec{r})=\sum_{k l}\left[\eta_{k l} e^{i \vec{G}_{k l} \cdot \vec{r}}+c . c .\right],
$$

where $\eta_{k l}$ are complex amplitudes, $\vec{G}_{k l}=k \vec{q}_{1}+l \vec{q}_{2}$, $\left(\overrightarrow{q_{1}}, \vec{q}_{2}\right)$ are the principal reciprocal lattice vectors and $k l$ are the Miller indices for a film of two dimensional triangular symmetry the reciprocal vectors are $\vec{q}_{1}=q_{c}(1,0)$ and $\vec{q}_{2}=q_{c}(-1, \sqrt{3}) / 2$, where $q_{c}=4 \pi / \sqrt{3} a_{c}$. For the commensurate $(\sqrt{3} \times \sqrt{3}) R 30^{\circ}$ phase, there are three equivalent sublattices with the lattice constant $a_{c}=\sqrt{3} a$ as shown in Fig. 1b. A given sublattice is a triangular lattice with lattice constant $a_{c}$ and displaced from the other sublattices by $\vec{\Delta}_{1}=[0,0], \vec{\Delta}_{2}=[a, 0]$ and $\vec{\Delta}_{3}=[2 a, 0]$.
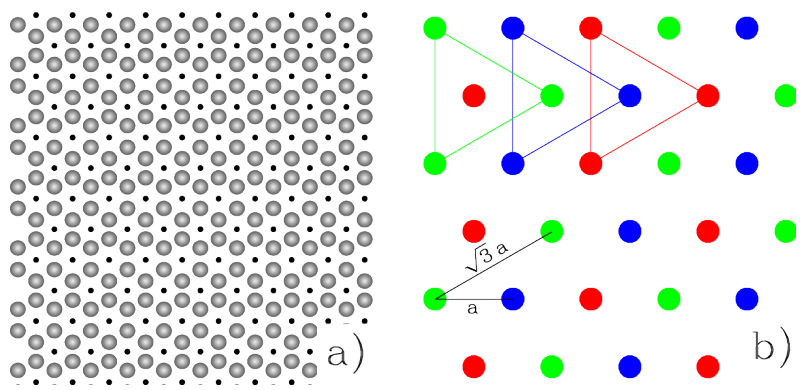

Fig. 1: Schematic of a) graphite surface and b) possible commensurate sublattices. In a) the grey circles and black points represent potential maxima and minima, respectively. In b) the red, blue and green points (or three shades of grey in monochrome rendering) represent the three equivalent $(\sqrt{3} \times \sqrt{3}) R 30^{\circ}$ commensurate sublattices.
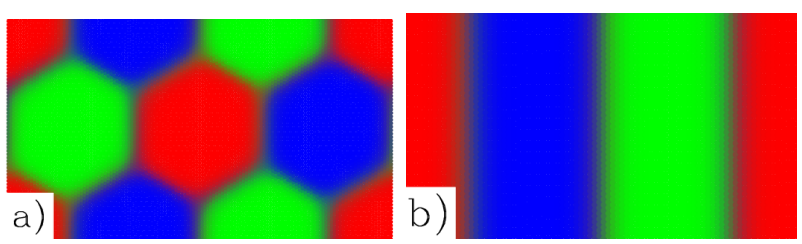

Fig. 2: The phases found numerically for $\epsilon=3 \%$. The colors correspond to the closest sublattice (shown in Fig. 1b) that the adsorbed atoms sit at for the a) HoI and b) SI $(\sqrt{3} \times \sqrt{3}) R 30^{\circ}$ phases.

Note that the potential for the $\mathrm{Xe} / \mathrm{Pt}(111)$ has exactly the same symmetry shown in Fig. 1 since the adsorption sites for this systems are the on-top positions for the $\mathrm{Pt}(111)$ substrate [1] with triangular symmetry, and the density field $n(\vec{r})$ for the $(\sqrt{3} \times \sqrt{3}) R 30^{\circ}$ phase of this system can be described by the same Fourier expansion as the $\mathrm{Kr} /$ Graphite and Xe/Graphite systems. Throughout the present study, we will adopt the simplest periodic adsorption potential with the right triangular symmetry containing the lowest-order harmonics as given by $V(\vec{r})=-V_{0} \sum_{k l} e^{i \vec{G}_{k l}^{s} \cdot \vec{r}}$, where $\vec{G}_{k l}^{s}$ are the same as $\vec{G}_{k l}$ given following Eq. (2) except rotated by $30^{\circ}$ and larger by a factor of $\sqrt{3}$. In the commensurate phase, the amplitudes, $\eta_{k l}$, in Eq. (2) are constant. To allow for the devel- 


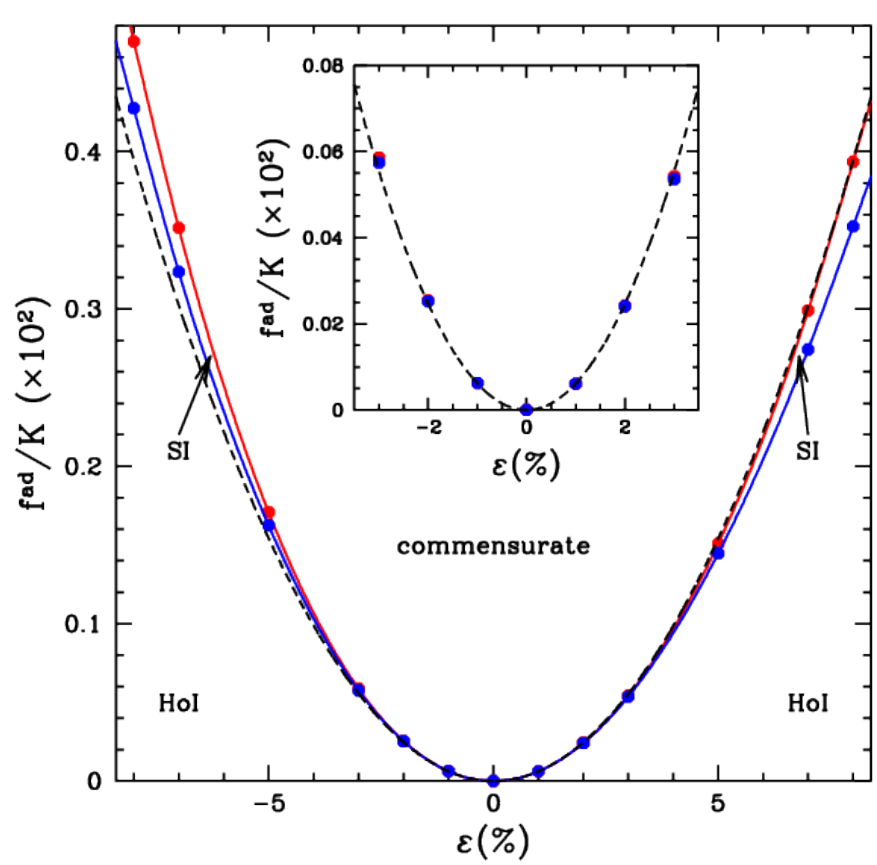

Fig. 3: Phase diagram from the effective single mode model, defined by Eq. (4). The red (upper) and blue (lower) points correspond to the C-SI and SI-HoI transitions, while the lines are guides the eye. The dashed black line corresponds to the Sine-Gordon prediction [31] for the C-SI transition, i.e., $f^{\text {ad }} / K=\left(\pi^{2} / 16\right) \epsilon^{2}$. Note in the inset the blue and red points almost overlap.

a free energy per unit area $A$ as,

$$
\begin{aligned}
F_{c}[\Phi, \Omega] / A & =F_{L}+3 \Delta B\left(\Omega^{2}+\Phi^{2}\right)-6 V_{0} \Omega \\
& +3 B_{x}\left(\Omega^{2}\left(q_{0}^{2}-3 q_{c}^{2}\right)^{2}+\Phi^{2}\left(q_{0}^{2}-q_{c}^{2}\right)^{2}\right) \\
& -4 t\left(\Omega^{3}+3 \Omega \Phi^{2}+\Phi^{3}\right) \\
& +v\left(\frac{45}{2} \Omega^{4}+90 \Omega^{2} \Phi^{2}+36 \Omega \Phi^{3}+\frac{45}{2} \Phi^{4}\right),
\end{aligned}
$$

where $F_{L}$ is a constant independent of $\Phi$ and $\Omega$. Note that in the $(\sqrt{3} \times \sqrt{3}) R 30^{\circ}$ phase, the adsorption potential $V(\vec{r})$ only couples to the second mode amplitude $\Omega$. Minimizing the free energy with respect to $\Omega$ first, we can solve for $\Omega$ as a function of $\Phi$. Substitution of this back into the free energy then yields a free energy functional $F(\Phi)$ that depends only on the amplitude of the first mode $\Phi$. This free energy describes a first order phase transition from a disordered or liquid state (i.e., $\Phi$ ) to an ordered or crystalline state ( $\Phi$ finite) as a function of $\Delta B$ as illustrated in Fig. 4. This procedure can obviously be generalized to eliminate

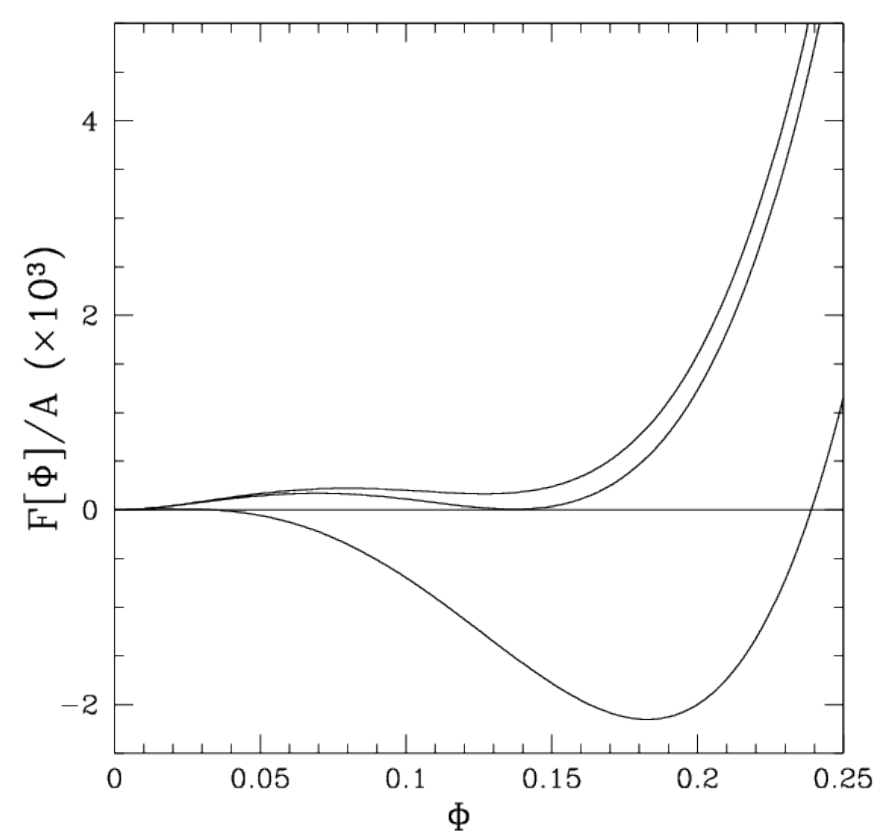

Fig. 4: Free energy density as a function of $\Phi$ for (from top to bottom) $\Delta B=0.05,0.047$ and 0.02 . For $\Delta B<0.047$ the free energy density is minimized by a crystalline state (finite $\Phi)$ and a liquid state for $\Delta B>0.047$.

all higher modes to yield an effective one-mode free energy $F(\Phi)$. Note that aside from the modification of all the coefficients of the various powers of the first mode amplitude $\Phi$ in the free energy, the crucial modification is in the coupling term to the adsorption potential, which in this case will yield nonlinear terms of the form $V_{0}\left(a \Phi^{2}+b \Phi^{3}+\ldots.\right)$.

Similar non-linear coupling terms would also appear if a non-linear coupling of the density, $n$, to the surface potential, $V$ were included in Eq. (1.) In this spirit we will 
consider the lowest-order non-linear coupling term that couples the first set of modes to the potential. Replacing $n V$ with $\lambda n^{2} V$ in Eq. (1) gives for the first set of amplitudes,

$$
\begin{aligned}
F_{\eta}= & \int d \vec{r}\left[\sum_{k l}\left(B^{x}\left|\mathcal{G}_{k l} \eta_{k l}\right|^{2}-\frac{3 v}{2}\left|\eta_{k l}\right|^{4}\right)\right. \\
& +\frac{\Delta B}{2} A^{2}+\frac{3 v}{4} A^{4}-2 t\left(\prod_{k l} \eta_{k l}+\text { c.c. }\right) \\
& \left.+2 \lambda V_{0}\left(\eta_{10} \eta_{01}+\eta_{01} \eta_{\overline{1} \overline{1}}+\eta_{\overline{1} \overline{1}} \eta_{10}+\text { c.c. }\right)\right],
\end{aligned}
$$

where the sums and products are over only the first set of modes, $A^{2} \equiv 2 \sum_{k l}\left|\eta_{k l}\right|^{2}, \mathcal{G}_{k l} \equiv \nabla^{2}+2 i \alpha \vec{G}_{k l}^{f} \cdot \vec{\nabla}+q_{0}^{2}-q_{c}^{2}$ and c.c. is the complex conjugate. As can be seen in the last line of Eq. (4) the density is now explicitly coupled to the potential, showing that this approach is an effective one-mode model for studying the $(\sqrt{3} \times \sqrt{3}) R 30^{\circ}$ phase and its transition into an incommensurate phase. In fact, all other similar higher-order commensurate states such as the $(\sqrt{7} \times \sqrt{7}) R 19.1^{\circ} p(2 \times 2)$ phases can be studied with a similar effective one-mode model, in spite of the much more complicated domain wall structures and larger number of equivalent sublattices [28].

In the commensurate $(\sqrt{3} \times \sqrt{3}) R 30^{\circ}$ phase, one of the three sublattices shown in Fig. 1b of the adsorption sites is occupied. As the amplitude of adsorption potential $V_{0}$ decreases and/or the misfit $\epsilon$ increases, the adsorbate system undergoes a transition to an incommensurate phase, consisting of commensurate regions occupying one of the sublattices, separated by domain walls. Depending on the values of the misfit parameter $\epsilon$ and the coupling strength of the adsorption potential $V_{0}$, such domain-wall structure can form a HoI phase (Fig. 2a) or a SI phase (Fig. 2b).

We have determined the phase diagram as a function of the lattice mismatch and strength of the adsorption potential by minimizing the free-energy functional using the effective single mode model of Eq. (4). The patterns emerge essentially due to a competition between the elastic and adhesion energies. For easy comparison with experiment we define the adhesion free energy per unit length, $f^{\text {ad }}$ as

$$
f^{\text {ad }}=f^{\text {com }}-f^{\text {elas }},
$$

where $f^{\text {com }}$ and $f^{\text {elas }}$ are the free energies of the commensurate and incommensurate states at the lattice spacing $a_{c}$ per unit area, respectively. For Eq. (4), $f^{\text {ad }}=8 \lambda V_{0} \Phi^{2}$. For the elastic contribution the relevant elastic modulus $K$ or a displacement from one sublattice to another is

$$
K=\left(C_{11}+C_{12}\right)^{2} / C_{11},
$$

which can be measured experimentally. For the free energy given in Eq. (4), $K=16 \Phi^{2} B^{x}$. Thus for easy comparison we plot the phase diagram in the plane of two dimensionless variables, the ratio of adhesion to elastic free energy densities $f^{\text {ad }} / K$ and the mismatch parameter $\varepsilon$. The result is shown in Fig. 3.
For a large misfit there is a SI phase between the commensurate and HoI phases. It can be shown [28] in the small-displacement limit that Eq. (4) can be mapped into a Sine-Gordon model for the C-SI transition (in the mean field limit) and a continuous transition occurs when $f^{\text {ad }} / K=\left(\pi^{2} / 16\right) \varepsilon^{2}$. As can be seen in Fig. 3 this prediction works very well at small strains. It appears that the C-SI and SI-HoI transition lines become very close at small strains, but to the limit of the numerical simulations they never merge indicating that the SI state region is present at all strains. It is important to note that it is very difficult to examine the strains less than $1 \%$ as the length scale of the patterns scale as the inverse of the misfit strain. For the smallest strain examined, $\varepsilon= \pm 1 \%$ the SI phase was present for some values of $f^{\text {ad }} / K$. Finally, we have also evaluated the phase diagram using the full PFC model of Eq. (1) without using the effective one-mode approximation and using the latter approximation with a $V n^{3}$ non-linear coupling instead of the $V n^{2}$ coupling used here. Although there are small quantitative changes in the location of the phase boundaries, the topology of the phase diagrams obtained from these models are essentially the same as that shown in Fig. 3 for the effective one mode model.

Close to the phase transition we found that for large misfit parameters, $|\epsilon|$, the free energy of the wall crossing is greater than that of the walls themselves. This can be inferred from the local free energy (i.e., the integrand of Eq. (4)) plot shown in Figs. 5a and b. For the case $\varepsilon=8 \%$ it can be seen that the free energy density is higher at the point where three domain walls cross. On the other hand, for lower misfits the free energy density reduces slightly at the crossing. For strong adsorption potential and large misfit, we find wide regions where the SI phase (Fig. 2 b)) is the lowest energy. Surprisingly, the SI to commensurate transition in this limit is of first order. For lower values of pinning the lowest energy phase is the HoI phase (Fig. 2 a)). The transition from SI to HoI is discontinuous. When the misfit parameter $|\epsilon|$ is decreased the stability range of the SI phase (Fig. 3) decreases quickly. For misfit parameters between $1 \%$ and $-1 \%$, it is numerically very costly to analyze the stability of the SI phase, but it reasonable to assume that the SI phase is present for all misfit parameters in the absence of fluctuations. The narrow region of the stability of the stripe phase in the phase diagram for decreasing misfit is consistent with the corresponding decrease of the wall crossing energy. In fact, according to the domain-wall arguments of Bak et al. [7], for any negative value of the domain wall crossing, a HoI structure would be favoured rather than a SI phase. Our results for the phase diagram imply that the commensurateincommensurate transition proceeds first via a transition from the commensurate phase into an SI phase and then from a SI phase into a HoI phase. For small misfit parameters and weak coupling to the substrate, the stripe phase occurs in such a narrow region of the phase diagram that it may not be experimentally observable. It should be kept 
in mind, however, that our results are at the level of mean field theory since it does not take into account the effects of thermal fluctuations, which may eliminate the SI phase.
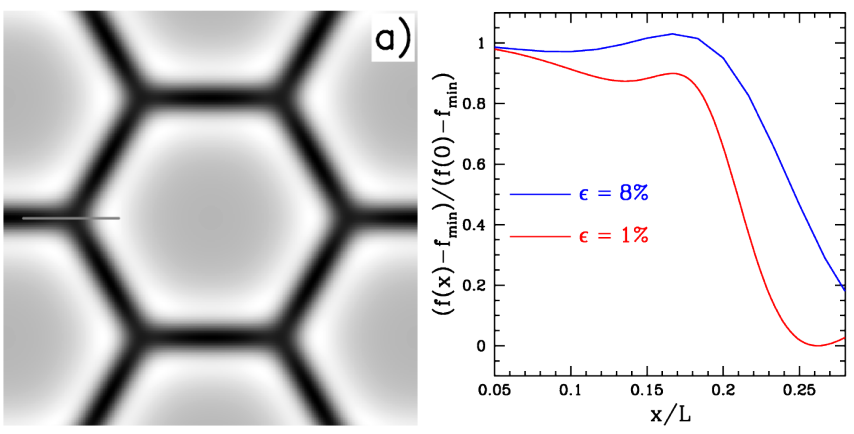

Fig. 5: In a) the local free energy density is displayed as a function of space for $\varepsilon=1 \%$ very close to the HoI/SI transition for a system of size $111 a_{f} \times 111 a_{f}$. In this figure the dark and light shades correspond to high and low free energy densities, respectively. In b) the normalized local free energy is plotted as function of $x$ along the grey line shown in a).

From the phase diagram of Fig. 3, the intermediate SI phase is more pronounced for large coupling parameter $f^{\text {ad }} / K$. For lower couplings, the SI phase exist only in a very narrow region. Experimentally it could appear as a direct transition from commensurate to HoI phase when the temperature and/or the coverage is varied. This behavior is consistent with the existing experimental data for Xe and $\mathrm{Kr}$ on Graphite [1]. Since for Xe/Pt(111) the corrugation potential is much larger, corresponding to a large coupling parameter, the transition from commensurate to HoI phase should clearly involve an intermediate SI phase, as observed experimentally $[1,5]$. Of course, for this interpretation to hold, we have to identify qualitatively the change of temperature as effectively changing the coupling parameter, and the change of coverage as effectively changing the mismatch parameter $\epsilon$.

The effective single mode model allows us to study the domain wall pattern in the incommensurate phase at large length scales, but still retaining atomistic resolution. One interesting question that can be addressed with this approach is the influence of adsorbate step edges on the orientation of the domain-wall structure. Recently, it has been observed experimentally by STM imaging of Xe overlayers on Graphite [27] that the domain walls orient perpendicularly to Xe island step edges on the surface. We can verify this behavior with the present model. To mimic a step edge on the overlayer, we set the pinning potential to a constant value on one side of the step edge and zero on the other size, and then follow the time development from an initial condition of a uniformly strained film (corresponding to the average strain of the equilibrium state) assuming non-conserved dissipative dynamics $[9,10]$, given by the equation of motion

$$
\frac{\partial \eta_{j}}{\partial t}=-\frac{\partial F_{\eta}}{\partial \eta_{j} *}
$$

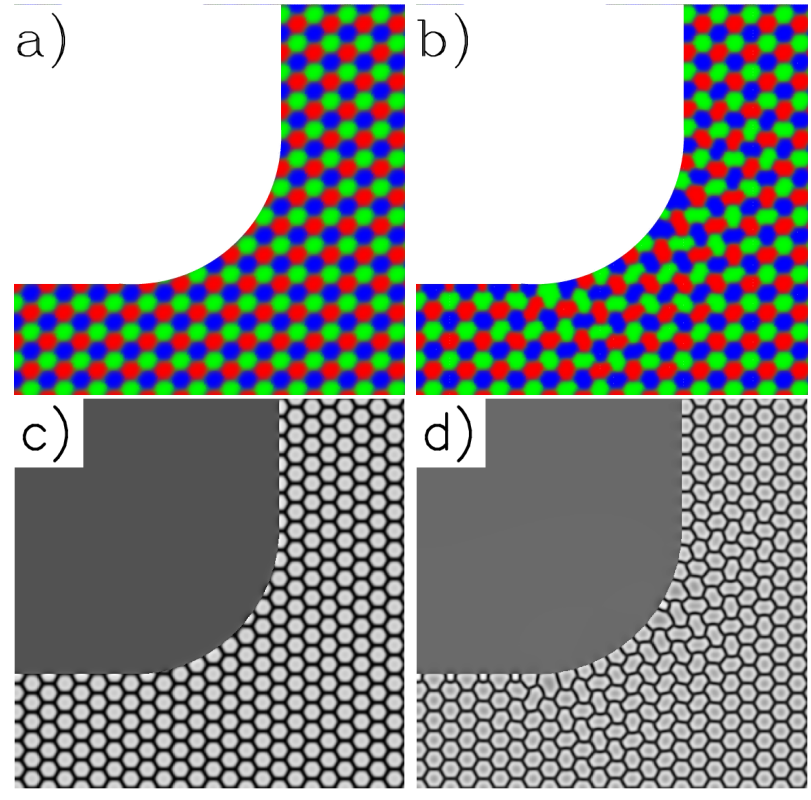

Fig. 6: Snapshots of a portion of a domain pattern at times $t=50$ in a) and c) and $t=50000$ in b) and d) for a strain of $\varepsilon=7 \%$. In a) and b) the colors correspond to the three sublattices and in c) and d) the color is proportional to the energy as in Fig. 5. Inside the central region $f^{\text {ad }}=0$ and $f^{\text {ad }}=0.27 \times 10^{-2}$ outside.

Fig. 6 shows the pattern in the HoI phase for increasing times near a step of a curved edge. The domain wall structure undergoes a reorientation near the step edge such that domain walls are preferentially perpendicular to the step edge, as observed experimentally [27].

In this work, we have introduced an effective one mode PFC model designed for studying higher order commensurate states such as the $(\sqrt{3} \times \sqrt{3}) R 30^{\circ}$ phase found in systems such as Xe/Pt(111), Xe/Graphite, and Kr/Graphite, and the corresponding phase diagrams as the temperature and/or lattice mismatch is varied. Our model allows us to study systems at macroscopic scales where the domain walls in the incommensurate phases can be separated over large distance scale. It also allows us to study the microscopic details of the domain wall structures both in the honeycomb incommensurate $(\mathrm{HoI})$ phase and the stripe incommensurate phase (SI) phases. In particular, we are able to evaluate the domain wall crossing energy as a function of the adsorption potential strength and lattice mismatch. New results such as the general persistence of a SI phase in between the commensurate phase and the HoI phase is found. Our results are qualitatively in agreement with the experimental observation where both the HoI and SI phases have been found in the Xe/Pt(111) system whereas only the HoI phase have been observed for the Xe/Graphite and $\mathrm{Kr} /$ Graphite systems. We have also found that near a step edge of the adsorbate, the domain walls tend to align perpendicular to the step edge, in agreement with recent experimental observation [27]. In 
the future, it would be interesting to follow up the present study with inclusion of thermal fluctuation effects. We have demonstrated before [30] that thermal fluctuations can be effectively incorporated in the PFC model by treating the PFC free energy functional as an effective Hamiltonian. It is known that thermal fluctuations can lead to generation of dislocations in the domain wall structure, possibly resulting in a fluid phase in between the commensurate and incommensurate phases [29].

$$
* * *
$$

We acknowledge the computational resources provided by the Aalto Science-IT project and the CSC IT Center for Science, Finland. This work was supported by the São Paulo Research Foundation (FAPESP, Grant No. 2014/15372-3) (E.G.), the CNPq (Brazi) (E.G.), the Watson Institute at Brown University under a Brazil Collaborative Grant (S.-C.Y.) and the National Science Foundation under Grant No. DMR-1506634 (K.R.E.) . CVA acknowledges financial support from CRHIAM-FONDAPCONICYT Project 15130015. T.A-N. acknowledges partial support from Aalto Science Institute and the Academy of Finland though grants nos. 251748 and 284621.

\section{REFERENCES}

[1] Bruch L. W., Diehl R. D. and Venables J. A., Rev. Mod. Phys, 79 (2007) 1381.

[2] Elder K. R., Chen Z., Elder K. L. M., Hirvonen P., Mkhonta S. K., Ying S.-Y., Granato E., Huang Z.-F. and Ala-Nissila T., J. Chem. Phys., 144 (2016) 174703.

[3] Suzanne J. and Gay J. M., Physical Structure - Handbook of Surface Science, edited by W. N. UnerTL, Vol. 1 (North-Holland) 1996, sect. Chapter 10 - The Structure of Physically Adsorbed Phases, p. 503-575.

[4] Specht E. D., Mak A., Peters C., Sutton M. , Birgeneau R. J., DAmico K. L., Moncton D. E., NAgler S. E. and Horn P. M., Z. Phys. B, 69 (1987) 347.

[5] Kern K., David R., Zeppenfeld P. and Comsa G., Surf. Sci., 185 (1988) 353.

[6] Bruch L. W. and Novaco A. D., Phys. Rev. B, 77 (2008) 125435.

[7] Bak P., Mukamel D., Villain J. and Wentowsaka K., Phys. Rev. B, 19 (1979) 1610.

[8] Villain J. and Gordon M. B., Surf. Sci., 125 (1983) 1.

[9] Elder K. R., Rossi G., Kanerva P., Sanches F., Ying S.-C., Granato E., Achim C. V. and Ala-Nissila T., Phys. Rev. Lett., 108 (2012) 226102.

[10] Elder K. R., Rossi G., Kanerva P., Sanches F., Ying S.-C., Granato E., Achim C. V. and Ala-Nissila T., Phys. Rev. B, 88 (2013) 075423.

[11] Elder K. R. and Grant M., Phys. Rev. E, 70 (2004) 051605.

[12] Goldenfeld N., Athreya B. P. and Dantzig J. A., Phys. Rev. E, 72 (2005) 020601.

[13] Athreya B. P., Goldenfeld N. and Dantzig J. A., Phys. Rev. E, 74 (2006) 011601.
[14] Athreya B. P., Goldenfeld N., Dantzig J. A., 382 Greenwood M. and Provatas M., Phys. Rev. E, 76383 (2007) 056706.

[15] Yeon D.-H., Huang Z.-F., Elder K. R. and ThornTON K., Phil. Mag., 90 (10) 237.

[16] Elder K. R., Huang Z.-F. and Provatas M., Phys. Rev. E, 81 (2010) 011602.

[17] Huang Z.-F., Elder K. R. and Provatas M., Phys. Rev. E, 81 (2010) 011602.

[18] Zhang Q. M., and Larese J. Z., Phys. Rev. B, 43 (1991) 938.

[19] Hakim T. M. and Glyde H. R., Phys. Rev. B, 37 (1988) 974.

[20] Kern K. and Comsa G., Phase transitions in surface films 2 -NATO ASI Series, Vol. 267 1991, p. 41 -65.

[21] Peters C. and Klein M. L., Phys. Rev. B, 32 (1985) 6077.

[22] Erbil A., Kortan A. R., Birgeneau R. J. and DresSelhaus M. S., Phys. Rev. B, 28 (1983) 6329.

[23] Moncton D. E., Axe J. D. and DiSalvo F. J., Phys. Rev. Lett., 34 (1975) 734.

[24] Moncton D. E., Axe J. D. and DiSalvo F. J., Phys. Rev. B, 16 (1975) 801.

[25] Wilson J. A., Disalvo F. J. and Mahajan S., $A d v$. Phys., 24 (1975) 117.

[26] Villain J., Surf. Sci., 98 (1980) 219.

[27] Grimm B., Hövel H., Bödecker M., Fieger K. and ReiHl B., Surf. Sci., 454 (2000) 618-622.

[28] Elder K. R. , unpublished, (2016) .

[29] Coppersmith S. N., Fisher D. S., Halperin B. I. , Lee P. A. and Brinkman W. F., Phys. Rev. Lett., 46 (1981) 549.

[30] Ramos J. A. P., Granato E., Achim C. V., Ying S.C., Elder K. R. and Ala-Nissila T., Phys. Rev. E, 78 (2008) 031109.

[31] Chaikin P.M. and Lubensky T. C. , Principles of condensed matter physics (Cambridge Press, Cambridge) 1995. 